\title{
The Value of Participating Scientist Programs to NASA's Planetary Science Division
}

\author{
Authors: \\ Louise M. Prockter, Johns Hopkins University Applied Physics Laboratory, Laurel, MD \\ (louise.prockter@,jhuapl.edu; 240-639-5042) \\ Meghan R. Wheeler, University of Virginia, Charlottesville, VA \\ Klaus-Michael Aye, University of Colorado, Boulder, CO \\ Kevin H. Baines, Jet Propulsion Laboratory, California Institute of Technology, Pasadena, CA \\ Michael T. Bland, United States Geological Survey, Flagstaff, AZ \\ David T. Blewett, Johns Hopkins University Applied Physics Laboratory, Laurel, MD \\ Serina Dineaga, Jet Propulsion Laboratory, California Institute of Technology, Pasadena, CA \\ Lori M. Feaga, University of Maryland, College Park, MD \\ Jeffrey R. Johnson, Johns Hopkins University Applied Physics Laboratory, Laurel, MD \\ Harry Y. McSween, University of Tennessee, Knoxville, TN \\ Clive R. Neal, University of Notre Dame, Notre Dame, IN \\ Carol S. Paty, University of Oregon, Eugene, OR \\ Julie S. Rathbun, Planetary Science Institute, Tucson, AZ \\ Britney E. Schmidt, Georgia Institute of Technology, Atlanta, GA \\ David B. Schwartz, Princeton University, Princeton, NJ \\ Janet A. Vertesi, Princeton University, Princeton, NJ

\section{Cosigners:} \\ Paul A. Abell (NASA/JSC) \\ Paul Byrne (NCSU) \\ Julie Castillo-Rogez (JPL) \\ Barbara A. Cohen (NASA/GSFC) \\ Kate Craft (JHU/APL) \\ Ingrid Daubar (Brown University) \\ Darby Dyer (PSI) \\ Carolyn M. Ernst (JHU/APL) \\ Justin Filiberto (JHU/APL) \\ Steven A. Hauck (Case Western U.) \\ Dana M. Hurley (JHU/APL) \\ Noam R. Izenberg (JHU/APL) \\ Natasha M. Johnson (NASA/GSFC) \\ Mallory Kinczyk (NSCU) \\ Robert T. Pappalardo (JPL) \\ Jani Radebaugh (Brigham Young) \\ Christina R. Richey (JPL) \\ Edgard Rivera-Valentin (LPI/USRA) \\ Andrew S. Rivkin (JHU/APL) \\ Linda Spilker (JPL) \\ Julie Stopar (LPI/USRA) \\ Elizabeth Turtle (JHU/APL) \\ Jennifer Whitten, (Tulane University) \\ Aileen Yingst (PSI)
}

Acknowledgments: We would like to offer our grateful thanks to the over 220 scientists and mission leaders in the planetary community who assisted with this study. 


\section{Executive Summary}

Participating Scientist programs provide significant value by increasing intellectual diversity within mission teams, enhancing the science return for NASA. Further, they increase demographic diversity, and open new and valuable pathways for mission participation. These programs should be included on every mission, whether competed or directed.

This white paper summarizes results presented in our more detailed community white paper published in May 2017 ([1]; hereafter referred to as Prockter et al.), which represented all of the NASA Analysis/Assessment groups. That study was conducted in response to perceived inconsistencies regarding the value of Participating Scientist (PS) programs to the NASA Planetary Science Division (PSD), mission leadership, and program participants. Through the use of detailed surveys, the study addressed three objectives: (1) Assess the value that PS programs add to NASA PSD missions; (2) Understand similarities and differences among existing and past programs, and gather lessons learned; and (3) Investigate how to maximize the usefulness of the programs for future missions, and provide useful, actionable feedback to NASA PSD. The recommendations from the Prockter et al. study are as valid today as they were three years ago, and we think it important that the value of Participating Scientist programs should be emphasized in the Planetary Science and Astrobiology Decadal Survey 2023-2032.

We found overwhelming support for PS programs from both the planetary science community and mission leadership. As expected, PS programs provide significant value in increasing intellectual diversity within a project science team, and hence enhance the mission's science return for NASA. Less obvious is that these programs also enhance demographic diversity within teams, and provide a valuable opportunity for many in the community who may not otherwise have access to mission participation. PS programs also provide useful career experience, training, and networking opportunities for participants, especially those in the early stages of their careers. As a result of our findings, we recommend that PS programs are included on every mission, regardless of whether competed or directed.

Survey results also demonstrated that the implementation of PS programs is inconsistent across NASA missions and that there is room for improvement. Recommendations include greater stability and transparency of PS funding; ensuring that PSs are added to mission teams as early as feasible; and providing more opportunities for team integration and mission operations experience.

Furthermore, commitment to non-US missions beyond funded instrument teams provides an excellent low-cost opportunity for science return and mission involvement, and NASA is encouraged to formulate a consistent policy for this type of mission involvement.

\section{Background and Approach}

The original Prockter et al. study, initiated during a February 2016 OPAG meeting, was motivated by discussions which demonstrated that NASA's views of the value of Participating Scientists might not be equivalent to those of Participating Scientists themselves, or of the leadership of missions on which they served. The study was carried out over the course of a year by a group of planetary community members which included Chairs or representatives from all six (at that time) Assessment/Analysis Groups (AGs).

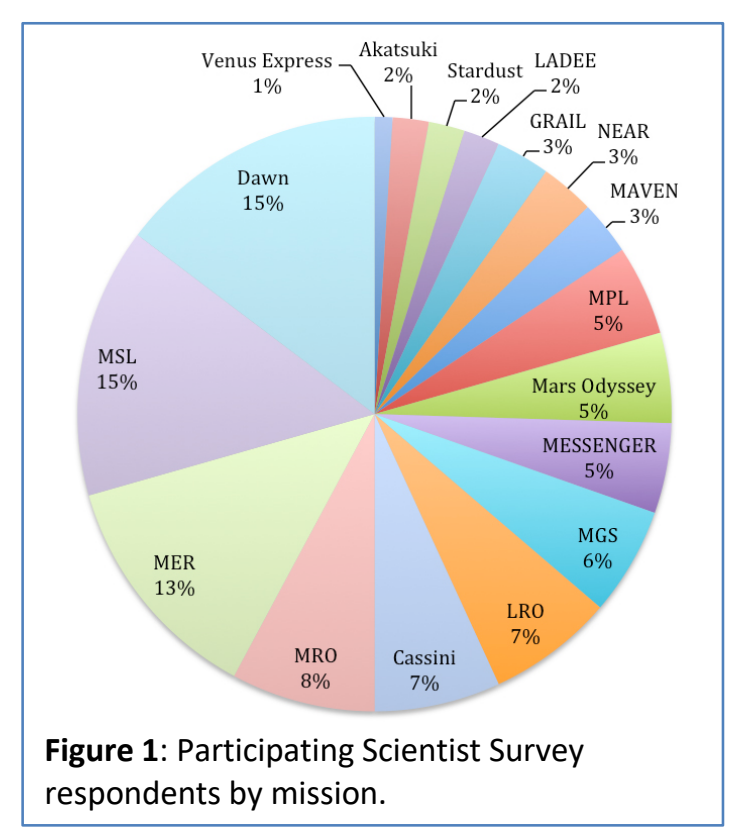


Our team also included three social scientists who were expert in the qualitative and statistical analysis of sociological data, to ensure that results were robust.

To meet the study objectives, two surveys were conducted, one of the planetary science community, and one of the leadership of twelve active and past planetary missions. The two groups were invited to share their experiences and opinions about PS programs, discussing what worked well, what could be improved, and what the perceived value was to them personally.

Survey 1 was for those who had served or were currently serving as PSs on planetary missions, as well as anyone who had an interest in, or opinion about these programs; Survey 2 was for leaders of planetary missions, either Principal Investigators and/or their deputies for competed missions, or Project Scientists and/or their deputies for directed missions. We defined the term "PS" to include both a scientist who is brought onto a mission after selection through a NASA Announcement of Opportunity (AO) PS call, and a Guest Investigator (GI), who is brought onto a mission through a NASA AO, primarily to carry out data analysis. We did not consider someone a PS if they were funded directly by the Project Office (PS's are funded by NASA HQ, not the project). Therefore, Co-Investigators, Collaborators, Postdocs, students, and Team Affiliates are not PSs according to our definition.

Study results were presented at meetings of OPAG, SBAG, VEXAG, MEPAG, the NASA Planetary Science Subcommittee, and the annual AAS Division of Planetary Sciences conference [2], and the white paper was sent to NASA PSD leadership and made widely available to the community. We received uniformly positive feedback on the white paper, including (informally) from NASA PSD program personnel and leadership.

\subsection{Survey 1: Current/former Participating Scientists and entire planetary community}

Survey 1 contained questions for existing/past PSs and/or anyone who had an interest in, or opinion about, these programs (Appendix A of Prockter et al., 2017). It was conducted anonymously, and distributed widely across the planetary community. The survey was divided into three parts:

a) Questions for past or current PSs (or GIs) about their experiences while participating in NASA planetary missions.

b) Questions for the entire community about their opinions on planetary missions and the perceived value of these programs to NASA.

c) A request to all respondents to a) and b) for limited demographic information, including age range and current career level.

Some of the questions in the community survey (Phase 1) asked for a simple yes/no answer, or for a number. We also asked for comments on respondents' experiences, and their opinions on the implementation and value of PS programs. Responses from both surveys were imported into the software package $n V i v o$ [3-4], a standard package for qualitative data analysis used by social science researchers that handles tabled entry and allows for flexible cross-correlation between types of responses. (A full description of the methodology used in analyzing the data from both phases of the survey is given in Appendix $C$ of Prockter et al., 2017.)

We received a total of 211 responses to the community survey, of which 101 met our criteria for current or former PSs or Guest Investigators (hereafter collectively referred to as PSs). As expected, the highest numbers of respondents (Fig. 1) were from currently or recently active missions (e.g., Dawn, MSL). High numbers of respondents also came from missions that had been active for a long time (e.g., MRO, MER). In general, responses appear consistent with the size and longevity of the mission, and the number of PSs that were funded by NASA. 


\subsection{Survey 2: Current/former planetary mission leadership}

Experiences of the authors of this paper led us to expect that mission leadership might have a different view of PS programs than the broader planetary community, given that they are managing large, complex projects and considerable thought went into assembling the core science teams. Opinions were solicited from mission leaders as to whether they viewed PS programs as useful additions to their projects, and to investigate the challenges in incorporating them. We therefore formulated a separate survey (Appendix B of Prockter et al., 2017) focusing on management challenges and lessons learned. Input for this survey was solicited in person or via email from the leaders of twelve recent and current missions, six of which were competed, and two of which did not have PS programs. Several of these had more than one PS call. Most were dedicated PS program calls although in one case, PSs were associated with a Data Analysis R\&A call. Although the leadership survey responses were not anonymous, we searched for generalities in the responses and removed information that identified specific missions.

\section{Discussion}

In general, comments from mission leadership and PSs reflected a consensus in regard to the overall value of the PS program for NASA (intellectual diversity allowing for an increase in science return and filling in gaps in expertise), the timing specifics of the program (bringing PSs on earlier can be beneficial), and funding (consistency in funding is desirable). We found no appreciable difference in the value of a PS program to NASA on competed vs. directed projects. From a personal perspective, many PSs commented about the value created by the program in advancing their career through networking and the opportunity to collaborate with other scientists and industry professionals, and by gaining mission experience in terms of data analysis and especially hands-on operational experience. This is significant, since career satisfaction is critical to retaining good people in any field.

One of the most significant results of the study is that PS programs draw from all career levels (Fig. 2). Almost one-third of PSs are within 7 years of their Ph.D. ("early-career" researchers, according to NASA), and half were within 10 years of their Ph.D. All but two of the $0-3$ year PSs were on directed missions; about half of those in the 10-20-year range served on competed missions, and the majority of the remainder served on directed missions (with Mars dominating the longer-term missions).

This range in career level suggests that PS programs may provide a significant opportunity for early-career researchers who may be at smaller institutions, or may not have associates on mission or proposal

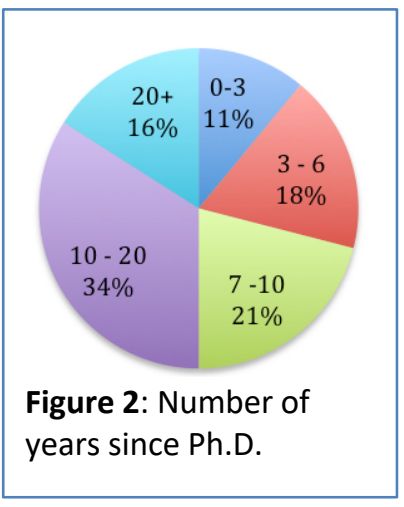
teams. Work by Rathbun et al. [5, 6] demonstrates that PS programs bring increased demographic diversity to planetary-mission science teams. They determined the percentage of women on science teams of robotic spacecraft missions and found that, for the past 15 years, the average percentage of women has remained flat at $\sim 15.8 \%$. They also determined the gender of scientists added to 9 science teams through PS and GI programs, finding that average percentage of women selected in these programs is $24.2 \%$. However, due to the small number of total selections in these programs, each individual call does not substantially increase the percentage of woman on the team. Only for those missions with multiple PS calls was the percentage of women in the overall team increased substantially (13.5\% - 25\% after two PS calls for MSL, for example). 


\section{Recommendations}

PS programs provide multiple benefits to missions and participants. Unless a mission is of such short duration that no clear value may be added by the addition of such a program, we recommend that: Participating Scientist programs should be included on every planetary mission, whether competed or directed. Detailed recommendations, and their justification follow:

\subsection{Planning of Participating Scientist programs}

The anticipated duration and scope of a PS program should be discussed between NASA HQ and the mission leadership in order to establish the needs of the program early on and to ensure that the program meets the needs of the mission as well as the planetary science community. This will help to ensure buy-in from mission leadership and will help them better prepare for integration of PSs onto their teams. We encourage mission leadership and NASA to discuss the scope of the PS programs with relevant community groups such as the Planetary Advisory Committee (PAC) and the NASA Analysis and Assessment Groups. We recommend that:

Expectations for the timing, duration, and scope of a Participating Scientist program should be agreed between NASA HQ and mission leadership as early as possible within the mission timeline, ideally during Phase A. The results of these discussions should be publicized to the planetary community through, e.g., NSPIRES, the Planetary Science Advisory Committee, relevant assessment and analysis groups, and community newsletters.

The sooner the planetary community learns of a PS program call - even if years in advance - the better prepared they can be to respond, and the quality of proposals responding to that call may improve accordingly. Furthermore, mission leadership should engage in clear communication with NASA about any expertise or capabilities that may be missing from their teams in order that the most valuable PSs can be selected for the team. We recommend that:

The planetary community should be given as much time as possible to prepare for a Participating Scientist call and sufficient information regarding mission payload and operations activities (e.g., through a Proposal Information Package), and existing team scientific capabilities and goals.

\subsection{Funding decisions}

With respect to funding, mission leadership generally noted that cost was a factor in deciding when to bring PSs onto a mission and that funding cuts limited the impact of the PS program. PSs generally accepted the necessary limitations of funding but noted a lack of consistency and transparency regarding funding scope, duration, and the requirement to repropose. Both groups made the overall point that funding issues and cuts negatively impacted the influence and impact of the PS program, and more funding could make the program even more valuable.

Given the widespread benefits of PSs programs to NASA and the planetary community, it is recommended that funding for these programs is identified as early as possible. If PS programs are included in all planetary missions, this could be at the AO phase for competed missions, and when directed missions are first put into the NASA budget. We recommend that

Sources of funding for Participating Scientist programs should be identified early, and ideally included in the release of a competitive mission $\mathrm{AO}$ or in the announcement of a directed mission.

While every attempt is made to hold to mission funding profiles, there are inevitably setbacks, and science funding is commonly a casualty of tightening mission budgets. Historically PIs may have been forced to choose between a PS program and some other significant need for their mission. 
Once the scope of a PS program is agreed between mission leadership and NASA HQ, the funds should be held as separate from other science-team funds unless there are significant developments in the mission that may warrant changes. Although funding is usually reduced in extended mission phases, this is an area where PSs can really contribute, and is an excellent time to train early-career scientists for mission work at a time when more senior scientists may be moving onto other projects. We therefore recommend that:

Once allocated, Participating Scientist funds should be held as inviolable, unless significant changes occur to the mission that would warrant a reduction in the program (e.g., failure of a portion of the mission or instrument), or an increase (e.g., groundbreaking findings that may require additional members with specific expertise). Participating Scientist programs should be included in extended mission phases.

There was frequently a lack of clarity regarding the timing and duration of the tenure of PSs. This issue would be partially mitigated if a clear PS program plan is put in place early in a mission timeline and is followed, but PSs should be made aware of the expectations of their funding, and whether/when they may need to repropose for another phase of the mission. We recommend that:

The amount of funding for a Participating Scientist program and expectations for its duration should be clearly communicated to Participating Scientists when they are selected.

\subsection{Timing of Participating Scientist additions}

Both mission leaders and the planetary community indicated that bringing on PSs earlier in a mission timeline was viewed as better, for the reason that PSs need time to familiarize themselves with mission operations and technicalities, and to socially integrate themselves into the existing team. However, there is no "one-size fits all" approach given that there is a wide disparity among mission classes and durations. Thus careful consideration is needed on a case-by-case basis to determine when is the best time to bring PSs onboard for maximum benefit.

PSs noted the benefits of getting involved in mission operations; operations involvement was also a contributor to the level of integration experienced within the existing team. Timing of selection is also a factor for PSs to be useful in operations.

Adding PSs for extended missions can have the benefit of expanding the mission team far beyond its original constituents, bringing in expertise that may be missing from the original science team but which is needed for new targets. Extended missions can also create opportunities for earlier-career researchers, including some who may not have even been in the field when the mission was selected. Even with fewer opportunities to get involved in mission design or operations, these later additions can still bring tremendous value to the mission science return. We recommend that:

Participating Scientists should be brought onto a mission as early as feasible, bearing in mind the trade between cost and integration issues. For most missions, the Participating Scientists should be brought onto a project at least one year before operations at the relevant major target. If possible (and appropriate), Participating Scientists should be given the opportunity to participate in mission operations.

\subsection{Team integration}

For PSs, the level of team integration had a strong impact on their program experience, however, mission leaders tended to focus more on the logistics of integrating PSs into a team. This indicates that mission leadership may not fully appreciate the influential impact of the integration experience for PSs and the effect it has on the productivity of the project itself. For PSs, it was clear that a positive team integration experience influenced their ability to work and have a meaningful 
contribution to the team, and more strikingly, that a bad team integration experience negatively impacted PSs and their experiences.

Once selected, PSs should be considered part of the science team, with the same status and access to data. Ensuring clear expectations for the timing and scope of PS programs early on in a mission's lifetime should help to facilitate this. Efforts should be made to help get the PSs up to speed as quickly as possible, to enable the PSs to fully participate in science discussions and data analysis, thereby enhancing the science return from the mission. We recommend that:

Full integration of Participating Scientists onto a project should be given high emphasis by mission leadership and the mission team. Expectations for the Participating Scientists' scope of work should be made clear when they join the team (e.g., operations, data analysis only, etc.) and they should be treated as equivalent to any other Co-Investigator on the team. This is especially important for Participating Scientists who are selected later in a mission (e.g., missions with a long cruise phase).

\section{PS program developments since release of original report}

Since our white paper was distributed in May 2017, NASA has offered a number of Participating Scientist programs. Their status as of August 2020 is shown in Table 1:

\begin{tabular}{|c|c|c|c|c|}
\hline Program & $\begin{array}{l}\text { Selection } \\
\text { month/ye } \\
\text { ar }\end{array}$ & $\begin{array}{l}\text { Number of } \\
\text { proposals }\end{array}$ & $\begin{array}{l}\text { Number of } \\
\text { selections }\end{array}$ & Notes \\
\hline $\begin{array}{l}\text { OSIRIS REx Participating } \\
\text { Scientists Program }\end{array}$ & Oct 2017 & 66 & 13 & \\
\hline New Horizons at MU69 & \multicolumn{4}{|c|}{ Not offered } \\
\hline $\begin{array}{l}\text { InSight Participating Scientist } \\
\text { Program }\end{array}$ & Aug 2018 & 68 & 22 & \\
\hline $\begin{array}{l}\text { Juno Participating Scientist } \\
\text { Program }\end{array}$ & $\begin{array}{l}\text { Apr \& Jun } \\
2019\end{array}$ & 34 & $3+9$ & $\begin{array}{l}\text { Second group selected only after finding } \\
\text { released by OPAG }\end{array}$ \\
\hline $\begin{array}{l}\text { Mars } 2020 \text { Returned Sample } \\
\text { Science Participating Scientist } \\
\text { Program }\end{array}$ & Sept 2019 & 54 & 10 & \\
\hline $\begin{array}{l}\text { BepiColombo Interdisciplinary } \\
\text { Scientists and Guest } \\
\text { Investigators program }\end{array}$ & Nov 2019 & $\begin{array}{l}19 \text { IDS from } \\
\text { US ( } 63 \% \text { of } \\
\text { total); } \\
8 \text { Gl from US } \\
\text { (76\% of total) }\end{array}$ & $\begin{array}{l}1 \text { IDS and } \\
2 \text { Gls from } \\
\text { US }\end{array}$ & $\begin{array}{l}\text { Selections made by ESA; unknown whether } \\
\text { numbers were limited by available NASA } \\
\text { funding. Prior to selection, max selection } \\
\text { rates stated by NASA as }<17 \% \text { IDS and } \\
<18 \% \text { GS }\end{array}$ \\
\hline $\begin{array}{l}\text { Akatsuki Participating Scientist } \\
\text { Program }\end{array}$ & Apr 2020 & 11 & 4 & \\
\hline $\begin{array}{l}\text { Mars } 2020 \text { Participating Scientist } \\
\text { Program }\end{array}$ & $\begin{array}{c}\text { Not yet } \\
\text { announce } \\
d\end{array}$ & Not known & $\mathrm{N} / \mathrm{A}$ & $\begin{array}{l}\text { Proposals reviewed but selections are } \\
\text { delayed (anecdotally due to lack of funding) }\end{array}$ \\
\hline $\begin{array}{l}\text { Korea Pathfinder Lunar Orbiter } \\
\text { Participating Scientist Program }\end{array}$ & $\begin{array}{l}\text { Expected } \\
\text { late } 2020\end{array}$ & Not known & $\mathrm{N} / \mathrm{A}$ & $\begin{array}{l}\text { Selections delayed due to delays in } \\
\text { mission; expected late } 2020\end{array}$ \\
\hline $\begin{array}{l}\text { DART Participating Scientist } \\
\text { Program }\end{array}$ & TBD 2021 & $\mathrm{~N} / \mathrm{A}$ & $\mathrm{N} / \mathrm{A}$ & Proposals are due 09/24/2020 \\
\hline $\begin{array}{l}\text { VIPER Participating Scientist } \\
\text { Program }\end{array}$ & TBD 2021 & $\mathrm{~N} / \mathrm{A}$ & $\mathrm{N} / \mathrm{A}$ & \\
\hline
\end{tabular}

Table 1: NASA PSD Participating Scientist calls and status since 2017. Information is from NSPIRES, other NASA documents, and personal communications. 
While NASA PSD has generally offered PS opportunities for its ongoing missions, there is still progress to be made. The Mars 2020 Participating Scientist selections are delayed (apparently due to a lack of funding), which will limit required mission operations training for selected PS's prior to the Feb. 18, 2021 landing. No PS program was offered for New Horizons, which carried out an encounter with the KBO MU69 on January 1, 2019 as part of its extended mission. This was despite ample time to offer a such a program, and the new small-body focus of the extended mission. The Juno PS call initially led to only three selections, and it was only after strongly worded findings from OPAG that additional PS slots were added. Nevertheless, this seems like a missed opportunity to enrich the Juno team with necessary satellite expertise during their extended mission.

One other area that could be strengthened is participation in international opportunities, which offers a relatively low-cost method of participation in non-US missions, and a great opportunity to US researchers. US Scientists have been supported by NASA on several international mission teams (e.g, Hayabusa2, Akatsuki), but funding and selection criteria are often opaque. In the case of the BepiColombo Interdisciplinary Scientists and Guest Investigators program, over two-thirds of the proposers were from the US (Table 1). Of the 19 US Interdisciplinary Scientist proposers only 1 was selected despite a possible 5 US slots.

One final unresolved issue is the status of US scientists who are participating on the JUICE mission. A number of US contributors were involved in European JUICE instrument proposals (and likely were a factor in their selection), but NASA has not committed any funds to these US CoIs (only the US instrument teams are funded). Despite OPAG findings and multiple queries to NASA, there is no resolution to this problem, and the US Co-Is continue to work in an unfunded capacity and without travel support.

We encourage NASA to continue to develop opportunities for US scientists to participate in non-US missions, and to develop policies which provide consistency and stable funding for these activities (e.g., [7]).

\section{References}

[1] Prockter, L.M, M. Aye, K. Baines, M. Bland, D. Blewett, S. Diniega, L. Feaga, J. Johnson, H. McSween, C. Neal, C. Paty, J. Rathbun, B. Schmidt (2017). The Value of Participating Scientist Programs to NASA's Planetary Science Division.

https://www.lpi.usra.edu/analysis/reports/Value of Participating_Scientist_Programs to_NASA_white paper final.pdf

[2] Prockter, L.M., M. Aye, K. Baines, M. Bland, D. Blewett, S. Diniega, L. Feaga, J. Johnson, H. McSween, C. Neal, C. Paty, J. Rathbun, B. Schmidt (2016). The Value of PSs on NASA Planetary Missions, AAS/Division for Planetary Sciences Meeting, 48, 332.02.

[3] NVivo qualitative data analysis Software; QSR International Pty Ltd. Version 11, 2016.

[4] Richards, Lyn. Using NVivo in Qualitative Research. London \& Los Angeles, Sage, 1999.

[5] Rathbun, J. A., J. Castillo-Rogez, S. Diniega, D. Hurley, M. New, R. Pappalardo, L. Prockter, K. Sayanagi, J. Schug, E. Turtle, A. Vasavada (2016). Historical Trends of Participation of Women Scientists in Robotic Spacecraft Mission Science Teams: Effect of Participating Scientist Programs, AAS/Division for Planetary Sciences Meeting, 48, 332.01.

[6] Rathbun, J. A., B.A Cohen, E.P. Turtle, J.A. Vertesi, A.S. Rivkin, S.M. Horst, M.S. Tiscareno, F. Marchis, M. Milazzo, S. Diniega, E.S. Lakdawalla, N. Zellner (2017). The Planetary Workforce: Goals through 2050, Planetary Science Vision 2050 Workshop, \# 8079.

[7] https://www.lpi.usra.edu/sbag/meetings/jun2020/FINAL_Findings_23.pdf 